\title{
Variación geográfica de las comunidades de parásitos de la merluza de tres aletas Micromesistius australis al sur de Sudamérica
}

\author{
Geographical variation of parasite communities in the southern blue whiting \\ Micromesistius australis around southern South America
}

\section{Mario George-Nascimento ${ }^{1}$, David Moscoso ${ }^{1}$, Edwin Niklitschek ${ }^{2,3}$ y Karen González $^{1}$}

\author{
${ }^{1}$ Departamento de Ecología Costera, Facultad de Ciencias, Universidad Católica de la Santísima Concepción, Casilla 297, \\ Concepción, Chile. mgeorgen@ucsc.cl \\ ${ }^{2}$ Centro i-mar, Universidad de los Lagos, Osorno, Chile. edwin.niklitschek@ulagos.cl \\ ${ }^{3}$ Centro Trapananda, Universidad Austral de Chile, Portales 73, Coyhaique, Chile
}

\begin{abstract}
A comparative analysis of the parasite fauna of the southern blue whiting Micromesistius australis was carried out on 165 specimens caught around the southern cone of South America in 4 fishing zones, three from the Pacific Ocean (South to Golfo de Penas, Diego Ramírez Islands and Navarino Island) and one from the Atlantic Ocean (Malvinas Islands). The goal was to detect possible population units or migratory patterns of the southern blue whiting. Fish samples from the Pacific Ocean were collected between October and December 2006 while those from Malvinas Islands were taken in December 2007. A total of 7,621 parasite individuals were collected and determined as members of 19 nominal taxa. The parasite fauna composition was similar between fishing areas but statistical analyses revealed significant differences in absolute and relative abundances of several taxa in infracommunities. After adjusting by the effect of host body size, it was found that the fishing zone had a significant effect on variations in total abundance, taxonomic richness, diversity and composition of parasite infracommunities. Some of these variations might correspond to the different local ecological scenarios in which the southern whiting inhabit around the southern cone of South America, and indicate that southern blue whiting stay and move differentially among fishing zones. These observations allow us to conclude that southern blue whiting do not constitute an ecologically homogeneous unit across the study area.
\end{abstract}

Key words: Population biology, fish resources, community ecology, Chile

\begin{abstract}
Resumen. - Se realizó un estudio comparativo del parasitismo de la merluza de tres aletas Micromesistius australis en el cono sur de Sudamérica, con 165 ej emplares provenientes de cuatro zonas de pesca, tres del Océano Pacífico (sur del Golfo de Penas, Islas Diego Ramírez e Isla Navarino) y una en el Océano Atlántico (Islas Malvinas). El objetivo fue detectar posibles unidades poblacionales o patrones migracionales de la merluza de tres aletas. Las muestras de peces del Océano Pacífico fueron tomadas entre octubre y diciembre del año 2006, en tanto que la del Océano Atlántico se tomó en diciembre de 2007. Se encontraron 7.621 individuos parásitos pertenecientes a un total de 19 taxa nominales. Los análisis mostraron diferencias significativas entre las zonas de pesca en abundancia absoluta y relativa de algunos taxa en las infracomunidades, aunque la composición de taxa parasitarios fue básicamente la misma. Luego de corregir por el efecto del tamaño corporal de los hospedadores, se encontró que la zona de pesca tenía un efecto significativo en las variaciones de la abundancia total, riqueza taxonómica, diversidad y composición de las infracomunidades. Algunas de estas variaciones pueden adjudicarse a las distintas condiciones ecológicas en que viven las merluzas de tres aletas en cada zona de pesca, y a su permanencia y movimiento diferencial en ellas, lo que permite concluir que no constituyen una unidad ecológicamente homogénea.
\end{abstract}

Palabras clave: Biología poblacional, recursos pesqueros, ecología de comunidades, Chile

\section{INTRODUCCIÓN}

La merluza de tres aletas Micromesistius australis Norman, 1937, es un pez de hábitos mesopelágicos y alta movilidad, relativamente longeva, y cuya distribución en el continente sudamericano abarca el cono sur del continente tanto en su vertiente Atlántica como del Océano Pacífico, ocupando un amplio espectro batimétrico. Los antecedentes de la biología poblacional de esta especie en Nueva Zelandia señalan que los adultos vuelven a desovar a sus lugares de origen, por lo que constituirían stocks diferentes (Hanchet 1999). En el Océano Pacífico sudoriental, sin embargo, los antecedentes son escasos, aunque un estudio reciente basado en una parte de las mismas muestras examinadas en este estudio y en el 
que se consideró sólo a los ejemplares entre 5 y 10 años de edad muestreados en las islas Malvinas y al sur del golfo de Penas, concluyó que en base al parasitismo y la microquímica de los otolitos, habrían dos subpoblaciones alrededor del cono sur de Sudamérica (Niklitschek et al. 2010).

El uso de los parásitos como indicadores de la biología de peces marinos ha adquirido creciente importancia ya que a través de su presencia o abundancia diferencial se puede deducir la existencia de unidades poblacionales, áreas de desove o rutas migratorias de sus hospedadores (MacKenzie 2002, MacKenzie et al. 2008). Los antecedentes parasitológicos para esta especie son escasos e incluyen el registro del monogeneo Diclidophora micromesisti y del myxozoo Kudoa alliaria (Suriano \& Martorelli 1984, Agnew et al. 2003).

En este estudio se comparan las características cualitativas y cuantitativas de los ensambles de taxa parasitarios que habitan en los individuos hospedadores entre cuatro zonas de pesca distribuidas en la vertiente Atlántica y Pacífica del cono sur de Sudamérica, incluyendo dos zonas de pesca en el extremo austral del continente sudamericano y un espectro más amplio de tamaños corporales del hospedador que los contemplados por Niklitschek et al. (2010). El objetivo es determinar si los parásitos pueden ser indicadores de estas zonas de pesca para lo cual se centró la atención en los taxa parasitarios que se acumulan en los hospedadores paraténicos o intermediarios, ya que usualmente poseen mayor tiempo de residencia en el hospedador y por lo tanto constituyen mejores indicadores de diferencias que involucran a un segmento mayor de la ontogenia del hospedador (Lester \& MacKenzie 2009).

\section{MATERIALES Y MÉTODOS}

En total se examinaron 165 ejemplares de Micromesistius australis provenientes del Océano Pacífico: 68 fueron del sur del Golfo de Penas ( $47^{\circ} 03^{\prime}$ S, $\left.75^{\circ} 04^{\prime} \mathrm{O}\right)$, y 11 de las

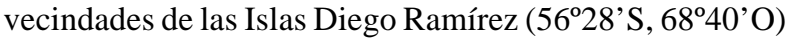

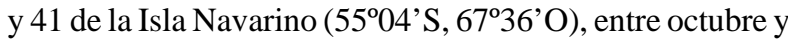
diciembre del año 2006, y del Océano Atlántico 45 ejemplares fueron obtenidas de las islas Malvinas ( $51^{\circ} 47^{\prime} \mathrm{S}$, $59^{\circ} 32^{\prime} \mathrm{O}$ ) en diciembre de 2007. A cada espécimen se le determinó la longitud a la horquilla $(\mathrm{LH}, \mathrm{cm})$ y fue examinado en sus parásitos.

Entre las variables parasitarias se determinó la abundancia para cada taxón, según zonas de pesca. Además, se registró la abundancia total, riqueza taxonómica, diversidad (índice de Brillouin), dominancia (índice de Berger-Parker), y composición de los ensambles de parásitos encontrados en cada individuo hospedador (= infracomunidades, ver Bush et al. 1997).

Luego de no alcanzar normalidad en la distribución de los datos de las variables parasitarias transformadas al $\log _{10}$ $(x+1)$, se usaron análisis de covarianza con las variables transformadas a su secuencia ordinal (ranking, Conover \& Iman 1982, Iman 1997). La significancia estadística de las diferencias en el tamaño corporal de los peces entre zonas de pesca fue evaluada mediante un análisis de la varianza de una vía. Se evaluó la asociación entre los descriptores parasitológicos y la longitud horquilla con el coeficiente de correlación de Spearman. En los análisis de la covarianza, la longitud horquilla fue la covariable y la zona de pesca fue el factor, y de ellos se excluyó a los taxa que poseían una prevalencia total inferior al 5\% en las zonas de pesca.

La composición de las infracomunidades fue resumida mediante una ordenación realizada con un análisis de correspondencia (AC) efectuado sobre la raíz cuadrada de la abundancia relativa de los taxa que se acumulan en los hospedadores (transformación de Hellinger, ver Legendre \& Gallagher, 2001), y usando el método de promediación recíproca (Jongman et al. 1995). Luego, se realizó un análisis de la varianza de los puntajes de las observaciones en el primer eje de la ordenación, con la zona de pesca como variable independiente (factor). En estos análisis se excluyó a los ectoparásitos y endoparásitos que utilizan a la merluza de tres aletas como hospedador definitivo, los que daban cuenta del $14,5 \%$ del total de parásitos recolectados. El $85,5 \%$ restante fueron los 12 taxa parasitarios que ocupan a la merluza de tres aletas como hospedador intermediario o paraténico, o que producirían infecciones de largo tiempo de residencia en el hospedador (e.g., Kudoa alliaria, quistes de etiología desconocida).

Otra manera de evaluar la eventual diferenciación de las infracomunidades entre las zonas de pesca consistió en el uso de un análisis discriminante no paramétrico, con un kernel cuyo radio fueron los tres vecinos más cercanos (Hand 1982). En estos análisis se consideró que las probabilidades de pertenecer a cada grupo eran proporcionales a su representación en el conjunto de grupos.

\section{Resultados}

La LH de los peces mostró diferencias significativas entre las zonas de pesca $\left(\mathrm{F}_{(3,161)}=5,07, P<0,002\right)$ ya que los ejemplares provenientes de Islas Malvinas poseían mayor LH que los recolectados al sur del golfo de Penas e Islas Diego Ramírez $(P<0,05)$, aunque los provenientes de Isla Navarino no se diferenciaban de los de las demás zonas de pesca $(P>0,05$, prueba de Tukey, Tabla 1$)$. 
Todos los peces estaban parasitados y en ellos se recolectaron 7.621 individuos parásitos pertenecientes a un total de 19 taxa nominales (Tabla 2). Todos los descriptores cuantitativos de las infracomunidades estaban correlacionados positivamente con la LH de Micromesistius australis (abundancia total, $\mathrm{r}_{\mathrm{s}}=0,54$, riqueza taxonómica, $r_{\mathrm{s}}=0,30$, diversidad, $r_{\mathrm{s}}=0,20, \mathrm{y}$ dominancia, $\mathrm{r}_{\mathrm{s}}=0,40, P<$ 0,05 en todos los casos, $n=165$ ). Además, la abundancia de Anisakis sp. $\left(\mathrm{r}_{\mathrm{s}}=0,75\right)$, Anisakidae gen. sp. $\left(\mathrm{r}_{\mathrm{s}}=0,31\right)$, Grillotia sp. $\left(r_{s}=0,27\right)$, Hemiuridae gen. sp. $\left(r_{s}=0,22\right)$, Diclidophora micromesisti $\left(\mathrm{r}_{\mathrm{s}}=0,19\right)$ y Kudoa alliaria $\left(\mathrm{r}_{\mathrm{s}}=\right.$ $0,30)$ mostraron coeficientes de correlación positivos con la longitud horquilla en el conjunto de los datos $(P<0,05, \mathrm{n}=$ 165 en todos los casos). Los demás taxa mostraron coeficientes de correlación no significativos con la LH ( $P>$ $0,05)$.

Tabla 1. Promedio y desviación estándar (entre paréntesis) de la longitud horquilla (LH, cm), abundancia total, riqueza taxonómica, diversidad y dominancia en las infracomunidades de parásitos en 165 ejemplares de Micromesistius australis provenientes de cuatro zonas de pesca. Las letras indican las diferencias entre parejas de medias ajustadas por la covariable / Mean and standard deviation (in parenthesis) of the fork length ( $\mathrm{LH}, \mathrm{cm}$ ), total abundance, taxonomic richness, diversity and dominance in parasite infracommunities found in 165 Micromesistius australis specimens collected at four fishing zones. Letters indicate significant differences between mean pairs when adjusted by the covariable

\begin{tabular}{lcccc}
\hline & $\begin{array}{c}\text { Sur del Golfo } \\
\text { de Penas } \\
\mathrm{n}=68\end{array}$ & $\begin{array}{c}\text { Islas Diego } \\
\text { Ramírez } \\
\mathrm{n}=11\end{array}$ & Isla Navarino & Islas Malvinas \\
\hline Longitud horquilla & $42,9(3,57)$ & $40,7(2,85)$ & $44,0(7,95)$ & $46,1(3,56)$ \\
Abundancia total & $56,9(53,9) \mathrm{a}$ & $17,54(7,76) \mathrm{b}$ & $47,14(79,49) \mathrm{b}$ & $36,04(31,54) \mathrm{b}$ \\
Riqueza & $5,84(1,63) \mathrm{a}$ & $4,82(1,83) \mathrm{a}$ & $4,97(2,02) \mathrm{b}$ & $5,31(1,53) \mathrm{b}$ \\
Diversidad & $1,56(0,42) \mathrm{a}$ & $1,30(0,46) \mathrm{a}$ & $1,35(0,46) \mathrm{b}$ & $1,43(0,38) \mathrm{b}$ \\
Dominancia & $0,25(0,14)$ & $0,24(0,15)$ & $0,24(0,18)$ & $0,29(0,15)$ \\
\hline
\end{tabular}

Tabla 2. Prevalencia ( $P$, en \%) y abundancia media ( $A$, desviación estándar entre paréntesis) de parásitos encontrados en 165 ejemplares de Micromesistius australis provenientes de cuatro zonas de pesca alrededor del cono sur de Sudamérica / Prevalence $(P$, in \%) and mean abundance ( $A$, standard deviation in parenthesis) of parasites in 165 Micromesistius australis specimens collected at four fishing zones around southern South America

\begin{tabular}{|c|c|c|c|c|c|c|c|c|}
\hline \multirow[b]{2}{*}{ Taxa Parasitarios } & \multicolumn{2}{|c|}{$\begin{array}{l}\text { Sur del Golfo de Penas } \\
\qquad n=68\end{array}$} & \multicolumn{2}{|c|}{$\begin{array}{c}\text { Islas Diego Ramírez } \\
n=11\end{array}$} & \multicolumn{2}{|c|}{$\begin{array}{c}\text { Isla Navarino } \\
\mathrm{n}=41\end{array}$} & \multicolumn{2}{|c|}{$\begin{array}{c}\text { Islas Malvinas } \\
\mathrm{n}=45\end{array}$} \\
\hline & $\mathrm{P}$ & $\mathrm{A}$ & $\mathrm{P}$ & A & $\mathrm{P}$ & A & $\mathrm{P}$ & A \\
\hline \multicolumn{9}{|l|}{ Ectoparásitos } \\
\hline Diclidophora micromesist $i$ & 58,8 & $1,93(2,29)$ & 63,6 & $2,09(2,39)$ & 65,8 & $2,41(2,77)$ & 82,2 & $3,73(4,61)$ \\
\hline Chondracanthus sp. & 8,9 & $0,24(0,90)$ & 0,0 & 0,00 & 2,4 & $0,02(0,16)$ & 2,2 & $0,02(0,15)$ \\
\hline \multicolumn{9}{|l|}{ Endoparásitos adultos } \\
\hline Ascarophis sp. & 16,2 & $0,62(2,17)$ & 72,7 & $4,09(6,59)$ & 61,0 & $7,10(12,06)$ & 4,4 & $0,04(0,21)$ \\
\hline Cucullanus sp. & 0,0 & 0,00 & 0,0 & 0,00 & 2,4 & $0,02(0,16)$ & 2,2 & $0,02(0,15)$ \\
\hline Acanthocephala indet. & 0,0 & 0,00 & 0,0 & 0,00 & 2,4 & $0,05(0,31)$ & 0,0 & 0,00 \\
\hline Derogenes sp. & 10,3 & $0,10(0,31)$ & 8,6 & $0,09(0,30)$ & 9,7 & $0,15(0,48)$ & 24,4 & $0,27(0,50)$ \\
\hline Hemiuridae gen. sp. & 16,2 & $0,51(1,68)$ & 18,2 & $0,54(1,51)$ & 43,9 & $1,54(2,71)$ & 31,1 & $3,29(19,18)$ \\
\hline Kudoa alliaria & 82,3 & $28,54(39,83)$ & 36,4 & $1,36(2,46)$ & 56,1 & $12,66(41,18)$ & 84,4 & $10,82(11,05)$ \\
\hline \multicolumn{9}{|l|}{ Endoparásitos larvales } \\
\hline Anisakis sp. & 98,5 & $13,01(12,51)$ & 100 & $6,55(5,99)$ & 87,8 & $19,12(36,16)$ & 97,8 & $14,04(8,59)$ \\
\hline Hysterothylacium $\mathrm{sp}$. & 80,1 & $3,38(3,12)$ & 36,4 & $0,55(0,82)$ & 26,8 & $0,76(2,01)$ & 82,2 & $1,87(1,56)$ \\
\hline Contracaecum $\mathrm{sp}$. & 73,5 & $1,91(2,11)$ & 45,4 & $0,64(0,81)$ & 48,8 & $1,68(2,91)$ & 24,4 & $0,40(0,84)$ \\
\hline Pseudoterranova sp. & 0,0 & 0,00 & 0,0 & 0,00 & 4,9 & $0,07(0,35)$ & 0,0 & 0,00 \\
\hline Anisakidae gen. sp. & 36,8 & $0,97(1,72)$ & 18,2 & $0,27(0,65)$ & 17,1 & $0,37(0,99)$ & 60,0 & $1,00(0,95)$ \\
\hline Hepatoxylon trichiuri & 73,5 & $4,66(5,63)$ & 36,4 & $0,45(0,69)$ & 21,9 & $0,29(0,60)$ & 24,4 & $0,38(0,78)$ \\
\hline Diphyllobothrium $\mathrm{sp}$. & 7,3 & $0,09(0,33)$ & 9,1 & $0,09(0,30)$ & 7,3 & $0,07(0,26)$ & 0,0 & 0,00 \\
\hline Pseudophyllidea fam. gen. sp. & 7,3 & $0,12(0,44)$ & 9,1 & $0,09(0,30)$ & 0,0 & 0,00 & 0,0 & 0,00 \\
\hline Grillotia sp. & 0,0 & 0,00 & 0,0 & 0,00 & 4,9 & $0,05(0,22)$ & 11,1 & $0,16(0,52)$ \\
\hline Corynosoma sp. & 7,3 & $0,50(3,52)$ & 0,0 & 0,00 & 12,2 & $0,12(0,33)$ & 0,0 & 0,00 \\
\hline \multicolumn{9}{|l|}{ Otros } \\
\hline Quistes de etiología desconocida & 5,9 & $0,37(1,98)$ & 27,3 & $0,73(1,27)$ & 21,9 & $0,66(2,00) \mathrm{a}$ & 0,0 & 0,00 \\
\hline
\end{tabular}

indet: indeterminado 
Al considerar los 6 taxa que mostraron correlaciones significativas de su abundancia con la LH de Micromesistius australis, se observaron diferencias significativas entre zonas de pesca en las pendientes de las regresiones sólo para el caso de Hemiuridae gen. sp. $\left(\mathrm{F}_{(3,157)}=4,40, P=0,0053\right)$. Estas diferencias consistían en que la pendiente era similar y positiva en Isla Navarino e Islas Malvinas, y no era distinta de cero en las otras dos zonas de pesca. En los demás taxa no había diferencias entre las pendientes de dichas regresiones, aunque la zona de pesca tenía un efecto significativo en las variaciones de la abundancia de varios taxa. En suma, había mayor abundancia de Hepatoxylon trichiuri $\left(\mathrm{F}_{(3,160)}=31,90, P<0,0001\right)$, Kudoa alliaria $\left(\mathrm{F}_{(3,}\right.$, $\left.{ }_{160)}=11,57, P<0,0001\right)$, Contracaecum sp. $\left(\mathrm{F}_{(3,160)}=12,87\right.$, $P<0,0001)$ e Hysterothylacium sp. $\left(\mathrm{F}_{(3,160)}=19,44, P<\right.$ $0,0001)$ en la muestra proveniente del sur del Golfo de Penas. En tanto, Ascarophis sp. $\left(\mathrm{F}_{(3,160)}=25,72, P<0,0001\right)$ y los quistes de etiología desconocida $\left(\mathrm{F}_{(3,160)}=5,83, P=0,0008\right)$ eran más abundantes en las Islas Diego Ramírez e Isla Navarino que en las otras dos zonas de pesca. Finalmente, las larvas de los nematodos Anisakidae gen. sp. $\left(\mathrm{F}_{(3,160)}=\right.$ $4,48, P=0,0048$ ) eran más abundantes al sur del Golfo de Penas y las Islas Malvinas que en las Islas Diego Ramírez.

Entre los descriptores cuantitativos de las infracomunidades, sólo la dominancia mostró diferencias significativas entre zonas de pesca en las pendientes de las regresiones con la longitud horquilla $\left(\mathrm{F}_{(3,157)}=4,35, P=\right.$ $0,0057)$, ya que eran mayores en las Islas Malvinas e Isla
Navarino que en las otras dos zonas de pesca. Luego de corregir por el efecto de la LH en los demás descriptores, se encontró que la zona de pesca tenía un efecto significativo en las variaciones de la abundancia total $\left(\mathrm{F}_{(3,160)}=12,22, P\right.$ $<0,0001)$, riqueza taxonómica $\left(\mathrm{F}_{(3,160)}=6,62, P=0,0003\right)$, diversidad $\left(\mathrm{F}_{(3,160)}=3,99, P=0,0089\right)$ y composición de las infracomunidades $\left(\mathrm{F}_{(3,160)}=23,09, P<0,0001\right.$, Tabla 1$)$. Éstas consistían en que los ejemplares provenientes del sur del Golfo de Penas albergaban infracomunidades de parásitos con mayor riqueza y diversidad que los provenientes de Isla Navarino o Islas Malvinas. La diferencia no fue significativa para el caso de las Islas Diego Ramírez. La abundancia total de parásitos en las infracomunidades era mayor en el sur del Golfo de Penas que en las otras zonas de pesca, y no había diferencias significativas entre las restantes.

En relación a la composición de las infracomunidades, el análisis de correspondencia mostró un valor propio $\lambda=$ 0,502 en el primer gradiente, cuya representación gráfica en función de la LH y de las zonas de pesca se muestra en Fig. 1. El análisis de la covarianza de los puntajes de las observaciones en el primer gradiente composicional indica que, luego de considerado el efecto de la $\mathrm{LH}$, la zona de pesca da cuenta del $58 \%$ de la varianza $(P<0,05)$. Finalmente, el análisis discriminante no paramétrico mostró una alta tasa de asignaciones correctas (74,5\%, Tabla 3), aunque para el caso de las Islas Diego Ramírez esto no se cumplió.

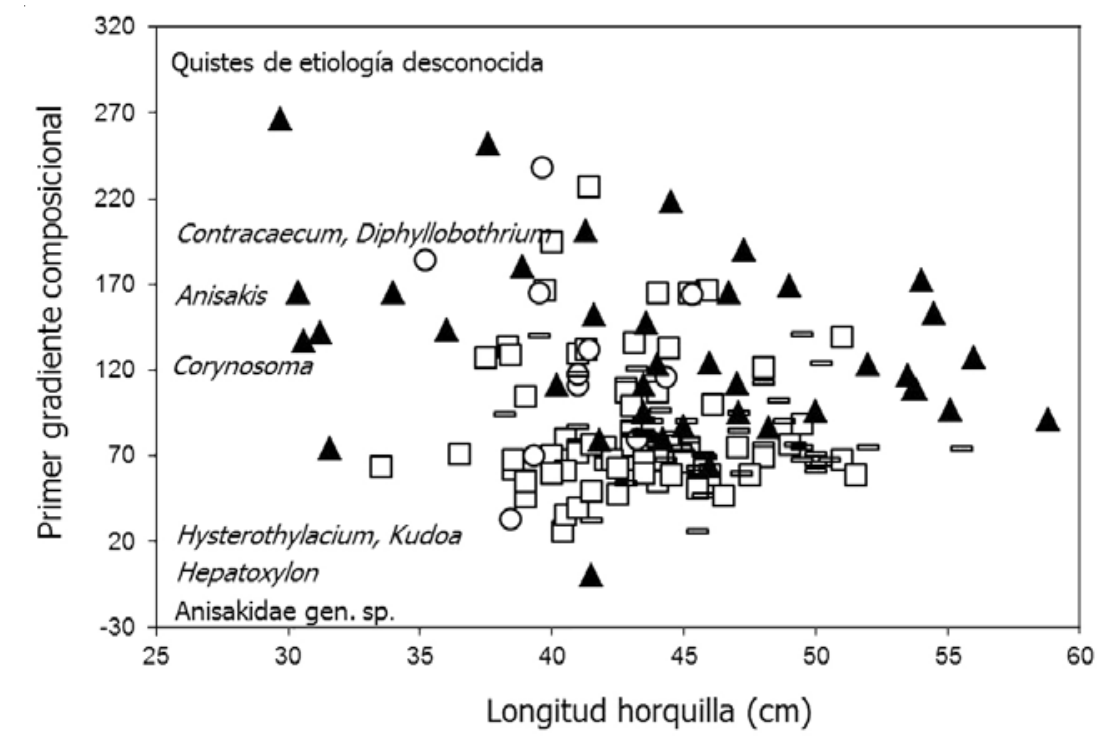

Figura 1. Relación entre el primer gradiente composicional de las infracomunidades de parásitos expresado en unidades de desviación estándar x 100, y la longitud horquilla de Micromesistius australis $(\mathrm{cm})$, según zona de pesca (círculo blanco: Islas Diego Ramírez, triángulo negro: Isla Navarino, cuadrado blanco: Sur Golfo de Penas, guión: Islas Malvinas) I Relationship between the first compositional gradient of parasite infracommunities expressed as standard deviation units $\times 100$, and the fork length $(\mathrm{cm})$ of Micromesistius australis, according to the fishing zone (white circle: Diego Ramírez Islands, black triangle: Isla Navarino, white square: South of Golfo de Penas, dash: Malvinas Islands) 
Tabla 3. Resultados de clasificación de un análisis discriminante no paramétrico de la composición de las infracomunidades de parásitos de Micromesistius australis, mostrando los porcentajes y números de ejemplares correctamente asignados a cuatro zonas de pesca / Classification results of a non-parametric discriminant analysis of the composition of parasite infracommunities in Micromesistius australis, showing percentage and number of specimens correctly assigned to four fishing zones

\begin{tabular}{lcccccc}
\hline & \% correcto & $\begin{array}{c}\text { Sur del Golfo } \\
\text { de Penas }\end{array}$ & $\begin{array}{c}\text { Islas Diego } \\
\text { Ramírez }\end{array}$ & $\begin{array}{c}\text { Isla } \\
\text { Navarino }\end{array}$ & $\begin{array}{c}\text { Islas } \\
\text { Malvinas }\end{array}$ & Total \\
\hline Sur del Golfo de Penas & 80,9 & 55 & 4 & 7 & 2 & 68 \\
Islas Diego Ramírez & 27,3 & 3 & 3 & 3 & 2 & 11 \\
Isla Navarino & 61,0 & 6 & 4 & 25 & 6 & 41 \\
Islas Malvinas & 88,9 & 5 & 0 & 0 & 40 & 45 \\
Total & 74,5 & 79 & 11 & 35 & 50 & 165 \\
\hline
\end{tabular}

\section{Discusión}

Aunque este estudio no es el primero que abarca la composición y variación geográfica del parasitismo de la merluza de tres aletas en la vertiente Pacífica y Atlántica del cono sur de Sudamérica, las muestras examinadas presentaron un mayor espectro de LH de los hospedadores (entre ca. 26 y $55 \mathrm{~cm}$ ) que los reportados por Niklitschek et al. (2010), lo que en parte permite inferir las modificaciones de las comunidades parasitarias en un rango más amplio de la ontogenia del hospedador. Además, se incorporaron dos zonas de pesca del extremo sur de Sudamérica que permiten un mejor discernimiento de lo que ocurriría con esta especie de hospedador en dicha zona. Sin embargo, cabe considerar que hay una creciente variedad de aproximaciones en estudios que utilizan a los parásitos como indicadores de la biología de sus hospedadores. En algunos, los investigadores seleccionan algunos taxa parasitarios como marcas, debido a su carácter acumulativo o largo promedio de vida en los hospedadores. Otros comparan las comunidades de parásitos (ya sea infracomunidades o comunidades componentes) entre zonas en estudio, sin hacer distinción por el tipo de parásito (e.g., Lester \& MacKenzie 2009 para una crítica). Además, entre estos últimos hay variedad de métodos empleados para el análisis de los datos, que motiva discusión (Perdiguero-Alonso et al. 2008, Pérez-del-Olmo et al. 2010). Ciertamente, los estudios parasitológicos que disponen información adicional de los hospedadores (morfometría, microquímica de otolitos, genética) son más completos pero no necesariamente más explicativos (MacKenzie et al. 2008). Se suma a lo anterior que recientemente se ha incorporado la genética de los parásitos como herramienta para discernir aspectos poblacionales de los hospedadores (e.g., Mattiucci et al. 2008).

La interpretación de los resultados de este estudio requiere considerar las características de la biología del hospedador y de los parásitos involucrados en las diferencias encontradas entre zonas de pesca (Lester \& MacKenzie 2009). Entre los ectoparásitos, no se encontraron diferencias notables en abundancia lo que sugiere que estos taxa se transmiten en forma homogénea entre sus hospedadores en el rango geográfico muestreado. Además, estos parásitos son de ciclo de vida directo por lo que se transmiten entre los peces mediante estados larvales de vida libre.

Entre los endoparásitos que alcanzan su estado adulto en Micromesistius australis, se destacó Ascarophis sp. por las diferencias encontradas en la abundancia entre las muestras más australes del Océano Pacífico (Islas Diego Ramírez e Isla Navarino) con la de la Islas Malvinas y con las del sur del Golfo de Penas. Además, se destacó la menor abundancia de Kudoa alliaria en las muestras provenientes de las Islas Diego Ramírez e Isla Navarino. Al respecto, los taxa parasitarios que alcanzan su madurez sexual en la merluza de tres aletas se transmiten mediante la ingestión de presas que albergan los estados larvales de parásitos (i.e., hospedadores intermediarios). En consecuencia, las diferencias encontradas responden a variaciones de la dieta de la merluza de tres aletas entre las zonas de pesca examinadas. En el caso particular de Ascarophis sp., las diferencias podrían asociarse a variaciones geográficas en el consumo de crustáceos responsables de su transmisión. En K. alliaria, las diferencias pueden deberse a variaciones geográficas en el consumo de poliquetos bentónicos, que serían sus hospedadores intermediarios, o a variaciones geográficas de la distribución de los hospedadores intermediarios o de los propios parásitos (Agnew et al. 2003). Sin embargo, las diferencias por este último parásito han de interpretarse como variaciones de más largo plazo porque su infección no desaparece durante la ontogenia del hospedador. Cabe considerar, que dado el tamaño y capacidad de movimiento de los hospedadores, los parásitos 
pudieron ser adquiridos en un lugar muy diferente del lugar en que fueron capturados. Esta observación es en parte coherente con lo registrado por Agnew et al. (2003) que sugieren una infección con K. alliaria antes de los dos años de edad, aunque la existencia de variaciones interanuales en la magnitud de este parasitismo no puede ser descartada.

Los taxa parasitarios que utilizan a la merluza de tres aletas como hospedador intermediario también se transmiten por la ingestión de presas infectadas con los estados larvales previos, y alcanzan su madurez sexual en los depredadores de Micromesistius australis, tales como peces teleósteos (e.g., Hysterothylacium sp.), elasmobranquios (Grillotia sp., Hepatoxylon sp.) y mamíferos (Anisakis sp., Contracaecum sp., Pseudoterranova sp., Diphyllobothrium sp., Pseudophyllidea gen. sp., Corynosoma sp.) o aves (Contracaecum sp. y Corynosoma sp.). De éstos parásitos los que mostraron mayores diferencias entre zonas de pesca, se destacan Hysterothylacium sp., Grillotia sp. y Hepatoxylon sp. Estas diferencias han de interpretarse en forma similar al caso de Kudoa alliaria, porque son infecciones de larga duración en el pez hospedador (Lester \& MacKenzie 2009). Finalmente, los quistes de etiología desconocida parecen ser más frecuentes en las Islas Diego Ramírez y en la Isla Navarino. Sin embargo, se desconoce su persistencia a lo largo de la ontogenia de M. australis.

En suma, las diferencias en el parasitismo que se observan entre las zonas de pesca pueden interpretarse como resultantes de las condiciones ecológicas en que viven las merluzas de tres aletas en las distintas zonas de pesca, y señalan que no constituyen una unidad ecológicamente homogénea, debido a que la merluza de tres aletas tendría permanencia diferencial en cada zona de pesca.

\section{LITERATURA CITADA}

Agnew DJ, TR Marlow, K Lorenzen, J Pompert, RC Wakeford \& GA Tingley. 2003. Influence of Drake Passage oceanography on the parasitic infection of individual year-classes of southern blue whiting Micromesistius australis. Marine Ecology Progress Series 254: 281-291.

Bush AO, KD Lafferty, JM Lotz \& AW Shostak. 1997. Parasitology meets ecology on its own terms: Margolis et al. revisited. Journal of Parasitology 83: 575-583.

Conover WJ \& RL Iman. 1982. Analysis of covariance using the rank transformation. Biometrics 38: 715-724.
Hanchet S. 1999. Stock structure of southern blue whiting (Micromesistius australis) in New Zealand waters. New Zealand Journal of Marine and Freshwater Research 33: 599-609.

Hand DJ. 1982. Kernel discriminant analysis, 264 pp, Research Studies Press, Letchworth, Herts.

Iman RL. 1997. A data-based approach to statistics, 848 pp, Duxbury Press, California.

Jongman RHG, CJF Ter Braak \& OFR van Tongeren. 1995. Data analysis in community and landscape ecology, 299 pp. Cambridge University Press, New York.

Legendre P \& ED Gallagher. 2001. Ecologically meaningful transformations for ordination of species data. Oecologia 129: 271-280.

Lester RJG \& K MacKenzie. 2009. The use and abuse of parasites as stock markers for fish. Fisheries Research 97: 1-2.

MacKenzie K. 2002. Parasites as biological tags in population studies of marine organisms: an update. Parasitology 124: S153-S163.

MacKenzie K, N Campbell, S Mattiucci, P Ramos, AL Pinto \& P Abaúnza. 2008. Parasites as biological tags for stock identification of Atlantic horse mackerel Trachurus trachurus L. Fisheries Research 89: 136-145.

Mattiucci S, V Farina, N Campbell, K MacKenzie, $\mathbf{P}$ Ramos, AL Pinto, P Abaúnza \& G Nascetti. 2008. Anisakis spp. larvae (Nematoda: Anisakidae) from Atlantic horse mackerel: Their genetic identification and use as biological tags for host stock characterization. Fisheries Research 89: 164-151.

Niklitschek EJ, DH Secor, P Toledo, A Lafon \& M GeorgeNascimento. 2010. Segregation of SE Pacific and SW Atlantic southern blue whiting stocks: integrating evidence from complementary otolith microchemistry and parasite assemblage approaches. Environmental Biology of Fishes 89: 399-413.

Perdiguero-Alonso D, FE Montero, A Kostadinova, JA Raga \& J Barrett. 2008. Random forests, a novel approach for discrimination of fish populations using parasites as biological tags. International Journal for Parasitology 38: 1425-1434.

Pérez-del-Olmo A, FE Montero, M Fernández, J Barrett, JA Raga \& A Kostadinova. 2010. Discrimination of fish populations using parasites: Random Forests on a 'predictable' host-parasite system. Parasitology 137: 18331847.

Suriano DM \& SM Martorelli. 1984. Monogeneos parásitos de peces pertenecientes al Orden Gadiformes de la plataforma del mar Argentino. Revista del Museo de La Plata, Zoología 13: 1-16.

Recibido el 3 de noviembre de 2010 y aceptado el 28 de enero de 2011 